\title{
The Design and Performance of Feedback Controllers for the Attenuation of Road Noise in Vehicles
}

\author{
Jordan Cheer and Stephen J. Elliott \\ Institute of Sound and Vibration Research, University of Southampton, Southampton, SO17 1BJ, UK
}

\begin{abstract}
(Received 15 February 2013; revised 15 October 2013; accepted 16 October 2013)
Active noise control systems offer a potential method of reducing the weight of acoustic treatments in vehicles and, therefore, of increasing fuel efficiency. The commercialisation of active noise control has not been widespread, however, partly due to the cost of implementation. This paper investigates the design and performance of feedback road noise control systems, which could be implemented cost-effectively by using the car audio loudspeakers as control sources and low-cost microphones as error sensors. Three feedback control systems are investigated, of increasing complexity: a single-input single-output (SISO) controller; a SISO controller employing weighted arrays of error sensors and control sources; and a fully-coupled multi-input multi-output (MIMO) controller. For each of the three controllers robustness and disturbance enhancement constraints are defined, and by formulating the three controllers, using an Internal Model Control (IMC) architecture and using frequency discretisation, the constrained optimization problems are solvable using sequential quadratic programming. The performance of the three controllers and the associated design methods are first evaluated in a simulated environment, which allows the physical limits on performance to be understood. Finally, to validate the results in the simulated environment, the performance of the three controllers has been calculated using data measured in a car cabin, and it has been shown that the fully-coupled MIMO controller is able to achieve significant low frequency road noise control, at the expense of increased implementation complexity compared to the SISO and SISO weighted transducer arrays feedback controllers.
\end{abstract}

\section{INTRODUCTION}

Noise in road vehicles is widely acknowledged as one of the key factors in governing their commercial success. ${ }^{1}$ Although passive acoustic treatments remain the dominant method of reducing both engine noise and road noise within the car cabin, ${ }^{2}$ there has also been considerable interest in active noise control measures for both of these types of noise. ${ }^{3}$ This interest has recently been driven by the need to improve the fuel efficiency of vehicles through the use of economical engine designs and by reducing the vehicle's weight. Economical engine designs such as variable displacement, which usually operates by deactivating a number of cylinders, often result in increased low frequency noise. Similarly, reducing the weight of a vehicle also results in increased low frequency noise. Low frequency noise is difficult to control using lightweight passive measures, and since active noise control systems are most effective at low frequencies and may be implemented within a car with relatively little increase in weight, they offer a convenient complementary solution. This is particularly true when the active noise control systems are integrated into the vehicle's electronic systems, for example, by employing the car audio loudspeakers. ${ }^{4}$

Low frequency engine noise has been successfully controlled using feedforward control systems employing an engine speed reference signal, low cost microphone error sensors, and the car audio loudspeakers as control sources. ${ }^{5}$ In a feedforward control system, as illustrated in Fig. 1(a), the control signal is generated by filtering the reference signal, $x$, which is correlated with the noise to be controlled, with filters that are adapted to minimise the error signals. A number of commer- cial feedforward engine noise control systems have been implemented due to their relatively low-cost. ${ }^{6-10}$ Reducing the weight of vehicles also increases the low frequency noise produced in the car cabin due to road-tyre interactions. Road noise has also been controlled using a feedforward control system, ${ }^{11}$ however, due to the random nature of road noise and the complex propagation path between structural excitation of the tyre and the acoustic noise produced in the car cabin, the implementation of a feedforward controller is significantly more demanding. Reference signals for a feedforward road noise control system have been obtained from accelerometers mounted to the vehicle's suspension and bodywork, ${ }^{11}$ however, in order to obtain sufficient coherence between the reference and disturbance signals it is necessary to employ at least six accelerometers. ${ }^{11,12}$ Although a feedforward control system has been reported to achieve A-weighted noise level reductions of up to $7 \mathrm{~dB}$ at the driver's ear position between 100 and $200 \mathrm{~Hz},{ }^{11}$ the need for multiple reference accelerometers means that the system is relatively expensive to implement and, therefore, has seen limited commercial implementation.

As a result of the high cost of a feedforward road noise control system, there is interest in implementing road noise cancellation using a feedback system, as this avoids the need for separate reference sensors. In a feedback control system, the control signals are produced by directly filtering the error signals, as shown in Fig. 1(b), which results in different performance characteristics compared to a feedforward controller. ${ }^{13,14}$ Feedback control of road noise has been the focus of research presented by Adachi and Sano, for example, ${ }^{14,15}$ which led to a mass-production system implemented in the 